\title{
A Intelligent Logistics Inventory Distribution Model Based On Pipeline Network And Ant Colony Algorithm
}

\author{
Jingwen $\mathrm{Li}^{1,2^{*}}$, Yifei Tang ${ }^{1}$ and Jianwu Jiang ${ }^{1}$ \\ ${ }^{1}$ Guilin University of Technology, Guilin 541004, China \\ ${ }^{2}$ Guangdong provincial land and resources surveying and Mapping Institute, \\ Guangzhou 510599, China
}

\begin{abstract}
With the popularization and application of emerging Internet technologies such as big data and cloud computing, the traditional B2B and B2C warehousing logistics management modes have not achieved synergy between various distribution stations and suppliers, achieving "one-to-one" means a distribution station is supplied by a manufacturer, and a customer is also supplied by a distribution station. The traditional logistics industry model can no longer meet the individual needs of customers. At present, the logistics industry has a series of problems such as slow delivery, slow turnover, high cost and poor service. Based on the theoretical basis of pipeline network and smart logistics, this paper proposes a pipeline network model of intelligent logistics, and improves the ant colony algorithm to improve transportation efficiency, which provides a guarantee for the efficient operation of the intelligent logistics platform.
\end{abstract}

\section{INTRODUCTION}

In recent years, China's logistics industry has grown rapidly, much higher than the growth rate of GDP during the same period. China has become the world's largest logistics market[1]. There are more than 700,000 large and small companies engaged in the logistics industry. In addition to the well-known postal and SF express companies, there are more than a dozen local courier companies in each city and county. This shows that the logistics industry is showing new vitality and potentiality[2]. However, while the logistics industry in China is developing rapidly, it also exposes a series of problems such as "scattering" of transportation resources, "chaos" of inventory resources, and "slow" logistics services. The traditional B2B and B2C models can no longer meet the development of the times[3]. "Intelligent logistics" is an important part of intelligent city construction. It bases on the modern logistics management model and introduces emerging Internet technologies, such as big data, cloud computing and GIS, to achieve unified management of all aspects of the logistics industry[4]. On the basis of "smart logistics", this paper introduces pipeline network model and ant colony algorithm to manage inventory and transportation links, implements strategies with higher service quality and more reasonable and effective transportation, so as to obtain better logistics benefits.

\section{Construction of Logistics Pipeline Network Inventory Model}

The pipeline network model is a digital simulation of the actual underground pipe network by computer[5]. It mainly consists of a network topology consisting of two elements: pipe segment and node. The pipe segment links two nodes. The pipeline network model expresses the structure properties, topological properties and hydraulic properties of the pipe and node.

\subsection{Objectified pipeline network model}

In the pipeline network model of this paper, the pipe segment is a two-dimensional object with only the left and right, front and rear directions, and the nodes are similar[6]. A node can connect several pipe segments, the inflow value of the node is positive[7], the outflow value is negative, and the two values are added to 0 :

$$
\sum_{i}^{n} q_{i}+Q_{i}=0, i=1,2,3,4 \ldots \ldots, n
$$

Where $q_{1}$ is the flow of the pipe into the node, $Q_{i}$ is the flow of the node into the pipe segment, and $n$ is the number of nodes in the pipeline network. The equation of the node is called the continuous flow equation of the pipeline network node. As shown in Figure 1, in the pipeline network model, the pipeline network diagram is generally used to represent the relationship between the research objects, such as the underground pipeline network. The nodes and pipe segments are respectively represented by points and lines, and a system is abstracted. Because the pipeline network model is applied to the intelligent logistics, this paper has modified the relevant attributes of the pipeline network.

For a certain pipeline network node set is expressed as: $\mathrm{V}=\left\{v_{1}, v_{2}, v_{3} \ldots, v_{n}\right\}$; pipe segment set: $\mathrm{E}=$

\footnotetext{
* Corresponding author: 451370648@qq.com
} 
$\left\{e_{1}, e_{2}, e_{3} \ldots, e_{n}\right\}$; the pipeline network model consists of these two combinations, remember It is: $\mathrm{G}=(\mathrm{V}, \mathrm{E})$, the pipe segment $e_{i}=\left(v_{j}, v_{k}\right)$ and the nodes $v_{j}, v_{k}$ are in communication with each other.

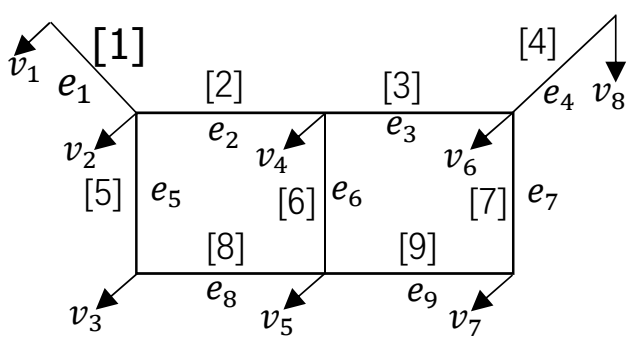

Fig. 1. Pipeline network model.

Referring to Figure 1, several attributes of the logistics pipeline network model can be obtained:

(1)Connectivity: Any two nodes in the pipeline network $G=(V, E)$ can be connected through other nodes and pipe segments. The two paths connecting the two nodes are multiple, indicating that different conditions can be selected if the conditions are different.

(2) Local directionality: In the pipeline network $\mathrm{G}=$ $(\mathrm{V}, \mathrm{E})$, the two nodes $v_{j}, v_{k}$ of the pipe segment $e_{i}=$ $\left(v_{j}, v_{k}\right)$ are ordered, and $e_{i} \neq\left(v_{j}, v_{k}\right)$. However, during transportation, for each segment (route), the flow of the connected nodes is bidirectional, and the pipe segmente $e_{i}=\left(v_{j}, v_{k}\right)=\left(v_{j}, v_{k}\right)$. The calculation of the flow rate of the pipe segment $e_{i}=\left(v_{j}, v_{k}\right)$ also does not satisfy the continuous flow equation. Only when the computational object is confined to a single transport process, the continuous flow equation of the node makes sense and therefore becomes locally directional.

(3) Non-constant: For the pipeline network $\mathrm{G}=$ $(\mathrm{V}, \mathrm{E})$, generally during the research process, the model will be simplified, and the speed and other properties in the pipe segment will not change with time, only one maximum value will be considered. However, in the transportation pipeline network model of this paper, factors such as the speed change during transportation and the time of staying at a node must be taken into consideration, which increases the complexity of the model.

\subsection{Inventory-based shunt model based on pipeline network}

In the traditional logistics industry, the distribution center forms a "one-to-one" relationship with customers and suppliers, which leads to waste of resources of other suppliers and distribution stations. Using the network model's connectivity and cloud computing and other related technologies to form a "cloud warehousing" model for information resource sharing, all logistics resources can be virtualized, including all resources of different suppliers and distribution points in different regions. This facilitates centralized management and unified scheduling. Moreover, these resources can be allocated according to the user's personalized require- ments, thereby realizing the management of resources in a "many-to-many" relationship.

Different distribution methods will result in different resource integration. When the resource integration of a certain distribution point is too high, the resources of other distribution points will inevitably be wasted, which is the defect of the traditional logistics distribution method. The customer's demand goes directly to the seller and supplier, and does not take into account the reasonable carrying capacity of the distribution node. When the bearing capacity of a node is not equal to its expected value, the greater the deviation, the slower the operating efficiency.
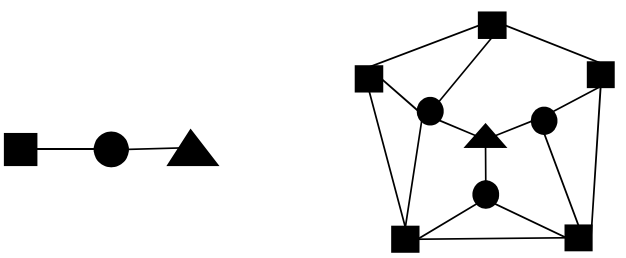

Busines

Distribution

client

Fig. 2. Traditional logistics model and Pipeline network intelligent logistics model.

For Figure.2, assume that the transport model $\mathrm{G}=$ $(\mathrm{V}, \mathrm{E}), \mathrm{c}$ is the customer point, $\mathrm{b}$ is the supplier, B is the supplier set, $\mathrm{d}$ is the distribution point, $\mathrm{D}$ is the distribution point set, and $\mathrm{C}$ is the customer's demand: For the traditional model, $\mathrm{C}$ refers to the supplier or manufacturer, and this paper proposes the pipeline network inventory diversion model, $\mathrm{C}$ is directed to the distribution site set $\mathrm{D}$, and then the site $\mathrm{D}$ presents the demand $Q_{d}$ to the supplier $\mathrm{B}$, and when each site $\mathrm{d}$ proposes the demand The relationship between $Q_{d}$ and its designed $Q$ distribution load is: when $Q_{d}=Q$, this is the best inventory situation; when $Q_{d}<Q$, the resources of the distribution station are not fully utilized, but the efficiency of distribution is not affected; when $Q_{d}>Q$ is used, the delivery station is already overloaded and the efficiency of delivery will be affected.

\section{Logistics Pipeline Network Inventory Model Based on Ant Colony Algorithm}

In the actual distribution process, from the supplier to the distribution site, the site to the customer, in addition to considering the load capacity of the site, there are other factors that affect the efficiency and cost of transportation. For the site collection $\mathrm{D}=$ $\left\{d_{1}, d_{2}, d_{3} \ldots d_{n}\right\}$, each of the sites $\mathrm{d}$ has different transportation efficiency and cost to the customer and the supplier, which is related to the location and weather of the site. The customer's demand is also one of the important factors in the construction of the distribution site. As customers increase, so does the demand and distribution points increase.

Aiming at the path problem between supplierdistribution point-customer, this paper proposes an improved ant colony algorithm to find the optimal path among them. The basic idea of the ant colony algorithm is to find the optimal feasible solution by using the ant to 
find the optimal path. All the nodes that the entire ant group passes constitute the optimal solution space. In the transport model $G=(V, E)$, assuming that there are $n$ ants and the total number of stations distributed is $\mathrm{m}$, then the probability that ant $\mathrm{k}$ selects the distribution node to be accessed is[8]:

$$
p_{i j}^{k}(t)=\left\{\begin{array}{c}
\frac{\tau_{i j}^{a}(t) \delta_{i j}^{\beta}(t)}{\sum \tau_{i r}^{a}(t) \delta_{i r}^{\beta}(t)}, j \in \text { allowed }_{k} \\
0, j \notin \text { allowed }_{k}
\end{array}\right.
$$

In Equation 2, allowed ${ }_{k}$ means the point at which ant $\mathrm{k}$ can traverse, and $\tau_{i j}(t)$ means the pheromone concentration of the distribution node. When $\alpha$ is larger, ant $\mathrm{k}$ tends to the path, $\delta_{i j}(t)$ is a distance heuristic information. When $\beta$ is large, the ant will increase the probability of selecting the current shorter path based on the greedy selection method. The pheromone $\alpha$ is updated when the ant $\mathrm{k}$ traverses the current node. The pipeline network model defined in this paper is quite different from the traditional ant system, so it cannot be directly solved by the ant colony algorithm. According to the characteristics of the pipeline network model, the following problems need to be redefined:(1)It is not necessary to traverse each node, and select the node that the ant needs to traverse according to the location of the distribution point;(2)The weight of each path will be subject to other factors, and there may be more than one node passing through;(3)The pipeline network has connectivity and local directionality, and does not need to form a closed loop.

In order to reduce the calculation time and increase the efficiency of calculation, the distribution points with higher distribution cost should be directly removed. This paper finds the optimal path, reduces the traditation cost, reduces the probability that the ant repeatedly shuttles in the path with lower weight, improves the transportation efficiency, and introduces the temporary variable $\omega_{i j}$ which is completely opposite to its function. When the ant passes the path composed of $\mathrm{i}, \mathrm{j}$ nodes, $\omega_{i j}$ will increase, and this variable is opposite to the action of pheromone $\alpha$. The larger the $\omega_{i j}$ is, the smaller the probability of passing the path. When the path is passed for the first time, the temporary variable $\omega_{i j}$ is calculated as:

$$
\omega_{i j}=d_{i j}\left(Q-Q_{d}+\varphi_{i j}(t)+v_{i j}(t)\right)
$$

In Equation 3, $d_{i j}$ represents the distance between the paths. If $Q_{d}<Q$ is the opposite, it means that the warehouse has reached the maximum load and does not traverse the change point. $\varphi_{i j}(t)$ represents the weather condition of the path. The larger the weather, the larger the value. , $v_{i j}(t)$ indicates the traffic situation between two points, the more the traffic is congested, the larger the value. As shown in Figure 3, the algorithm flow for optimal path optimization using the improved ant colony algorithm is:

Step1: Initialization data: Temporary variable $\omega_{i j}$ current cycle number $N_{c}$, maximum cycle number $N_{\max }$ according to formula 3 .

Step2: Assign ants evenly to individual suppliers.
Step3: Calculate the probability of the next distribution point based on the temporary variable $\omega_{i j}$ and Equation 2 and transfer.

Step4: Determine whether the delivery point, the customer has completely traversed, and if so, update the current optimal path, update the pheromone, the number of cycles $N_{c}=N_{c}+1$, and continue to the next step; otherwise, update the temporary variable, ant $\mathrm{k}=\mathrm{k}+1$, return step2.

Step5: If $N_{c}<N_{\max }, N_{c}=N_{c}+1$ and return step2, otherwise the optimal path is output.

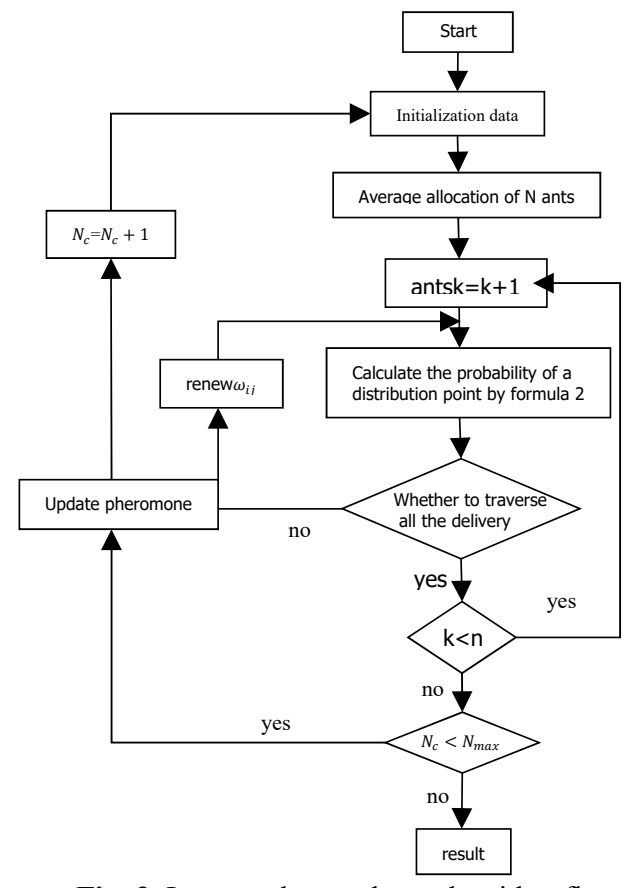

Fig. 3. Improved ant colony algorithm flow.

\section{Conclusion}

This paper studies and analyzes the advantages of the pipeline network model, and combines the characteristics of smart logistics to establish a smart logistics pipeline network model. In the pipeline network model of smart logistics, the "many-to-many" relationship between suppliers, distribution points and customers is formed, and the optimal inventory quantity of each distribution point is established. Based on the model, an improved ant colony algorithm is proposed to maximize the total transportation efficiency, reduce the logistics cost and improve the quality of logistics service.

\section{Foundation items:}

1. Development and Application of Intelligent Security Early Warning System for Visitors in Smart Scenic Areas and Time-Space Virtual Reconstruction Technology Item Number: 20170220

2. Research on Intelligent Indoor Positioning Method Based on WIFI and Its Application

Item Number: 2018-B-02 


\section{References}

1. He Liming. Development Trend of China's Smart Logistics.J.China Circulation Economy, 31(06):37,2017.

2. Wang Xinyue.Study on the Development of China's Smart Logistics and Countermeasures.J.Railway Transport and Economy, 39(04):37-41,2017.

3. Wang Yazhou. Research and implementation of scenic intelligent tour guide service system based on Android platform(Shenyang Institute of Technology, 2017).

4. Wang Xianmei. Research on the theory, method and application of smart cloud logistics based on big data(Zhejiang University of Science and Technology, 2015).

5. Zhang Chunxia,Peng Donghua.The Countermeasures of China's Smart Logistics Development.J.China Circulation Economy,2013,27(10):3539.

6. Wang $\mathrm{Xu}$, Tian Shuai Hui, Wang Zhenfeng, Xing Lebin. Dynamic logistics service network operation mechanism and distribution of interests .J. East China economic management, 2011,25 (05): 115118.

7. Ho Yang. New method of hydraulic model for urban drainage network and energy saving technology of pumping station(Hangzhou Dianzi University, 2011) .

8. Xu Feng, Du Junping. Application of improved ant colony algorithm in tourism route planning.J. computer engineering and application, 2009,45 (23): 193-195+226. 